\title{
Aberrant STYKI expression in ovarian cancer tissues and cell lines
}

\author{
Kesmic A Jackson ${ }^{1}$, Gabriela Oprea ${ }^{2}$, Jeffrey Handy ${ }^{3}$ and K Sean Kimbro*1
}

Address: ${ }^{1}$ Department of Hematology and Medical Oncology, Winship Cancer Institute, Emory University School of Medicine, Building C, Room C4090, Atlanta, GA 30322, USA, ${ }^{2}$ Pathology and Laboratory Medicine, Emory University School of Medicine, Atlanta, GA, USA and ${ }^{3}$ Division of Digestive Diseases, Emory University School of Medicine, Atlanta, GA, USA

Email: Kesmic A Jackson - biojak@langate.gsu.edu; Gabriela Oprea - goprea@emory.edu; Jeffrey Handy - jhandy@emory.edu; K Sean Kimbro* - kskimbr@emory.edu

* Corresponding author

Published: 21 October 2009

Journal of Ovarian Research 2009, 2:15 doi:10.1186/1757-2215-2-15
Received: 31 August 2009

Accepted: 21 October 2009

This article is available from: http://www.ovarianresearch.com/content/2/1/15

(c) 2009 Jackson et al; licensee BioMed Central Ltd.

This is an Open Access article distributed under the terms of the Creative Commons Attribution License (http://creativecommons.org/licenses/by/2.0), which permits unrestricted use, distribution, and reproduction in any medium, provided the original work is properly cited.

\begin{abstract}
Background: Overexpression of STYKI, a putative serine/threonine and tyrosine receptor protein kinase has been shown to confer tumorigenicity and metastatic potential to normal cells injected into nude mice. Mutation of a tyrosine residue in the catalytic STYKI domain attenuates the tumorigenic potential of tumor cells in vivo, collectively, suggesting an oncogenic role for STYKI.
\end{abstract}

Methods: To investigate the role of STYKI expression in ovarian cancer, a panel of normal, benign, and ovarian cancer tissues was evaluated for STYKI immunoreactivity using STYKI antibodies. In addition, mRNA levels were measured by reverse transcription PCR and real-time PCR of estrogen receptors, GPR30 and STYKI following treatment of ovarian cell lines with estrogen or GI, a GPR30 agonist, as well as western analysis.

Results: Our data showed higher expression of STYKI in cancer tissues versus normal or benign. Only normal or benign, and one cancer tissue were STYKI-negative. Moreover, benign and ovarian cancer cell lines expressed STYKI as determined by RT-PCR. Estradiol treatment of these cells resulted in up- and down-regulation of STYKI despite estrogen receptor status; whereas G-I, a GPR30-specific agonist, increased STYKI mRNA levels higher than that of estradiol.

Conclusion: We conclude that $S T Y K I$ is expressed in ovarian cancer and is regulated by estrogen through a GPR30 hormone-signaling pathway, to the exclusion of estrogen receptor-alpha.

\section{Introduction}

Ovarian cancer causes more deaths in women than any other gynecological cancer. The number of deaths caused by ovarian cancer is exacerbated by the lack of reliable screening, specific symptoms, and effective treatments. The National Cancer Institute estimates that 21,550 new cases of ovarian cancer will be diagnosed in the US in 2009. Women diagnosed with localized, regional, and distant ovarian cancer have a 93\%, 69\%, and 30\% 5-year survival rate, respectively [1-3]. However, diagnosis of localized ovarian cancer only occurs in about $19 \%$ of the cases due to a lack of reliable screening techniques and the absence of specific symptoms.

Ovarian cancer samples overexpress a putative serine-threonine receptor protein kinase, STYK1, as demonstrated by 
microarray analysis [4]. The human STYK1 kinase domain shares approximately 30-34\% identity with FGFR (fibroblast growth factor receptor)/PDGFR (platelet-derived growth factor) family members, which have been shown to function as oncogenes [5]. STYK1 overexpression constitutively activated the RAS/MAPK, STAT1, and STAT3 pathways in NIH3T3 cells [6]. Interestingly, ovarian cancer cells were shown to constitutively express high levels of STAT3 [7,8]. Furthermore, BaF3 cell lines overexpressing STYK1 proliferated in media without serum or growth factors. Inoculation of these cells into nude mice induced tumor formation within one week and the cells metastasized after 4 weeks. Introducing a tyrosine to phenylalanine point mutation into the catalytic domain of STYK1 blocked cell proliferation as well as STYK1-induced tumorigenesis [6,9]. STYK1 expression is regulated by estrogen in $\mathrm{ER} \alpha$ (estrogen receptor alpha)-negative (MDA-MB231) and ER $\alpha$-positive MCF7) breast cancer cells based on microarray analysis and real-time PCR analysis [10].

Estrogen receptors play a critical role in ovarian tumor cell growth. Ovarian surface epithelial cells produce estradiol and estrone, and the ovary is a key target of estrogen [11]. The postmenopausal ovary produces little or no estrogen; conversely, increased steroid hormone levels have been observed in the plasma of ovarian cancer patients [12]. The occurrence of ovarian cancer increases dramatically in menopausal women. Furthermore, previous studies report a correlation between plasma estradiol, progesterone, and androstenedione with stage of disease $[13,14]$. However, the mechanisms by which estrogen receptors contribute to ovarian tumorigenesis are still unclear [4]. GPR30, a novel estrogen receptor, and ER $\alpha$ stimulation by both G-1 (GPR30-specific ligand) and estradiol were shown to synergistically induce proliferation of breast and ovarian cancer lines [15].

In this study we examined STYK1 immunoreactivity in normal, benign, and malignant ovarian tissues. To investigate the role of estrogen and GPR30 in STYK1 regulation, we treated a benign and several malignant ovarian cancer cell lines with estradiol and G-1. We describe differences in STYK1 RNA and protein expression levels in treated versus untreated ovarian tumor cells. We also compare estradiol- and G-1-induced STYK1 expression. In the present report, we show that STYK1 expression is associated with ovarian tumorigenesis. Furthermore, we provide evidence for estrogen-mediated STYK1 regulation through an unknown GPR30 signaling pathway.

\section{Materials and methods Chemicals}

$17 \beta$-estradiol and BSA-conjugated estradiol were purchased from Sigma-Aldrich (Sigma, St. Louis, MO). 1-(4-
(6-Bromobenzo[1,3]dioxol-5-yl)-3a, 4,5,9b-tetrahydro$3 \mathrm{H}$-cyclopenta [c]quinolin-8-yl)-ethanone (G-1) was purchased from Calbiochem (San Diego, CA).

\section{Antibodies}

STYK1 and GPR30 antibodies were purchased from AbCam (Cambridge, MA). $\alpha$-Tubulin antibody was purchased from Millipore (Billerica, MA).

\section{Cell culture}

HS832, OvCar3, and CaOv3 were obtained from American Type Culture Collection (Manassas, VA). SkOv3, OvCar5, OvCar8, and IGROV1 were kindly provided by the lab of Dr. Neil Sidell (Emory University School of Medicine, Department of Gynecology and Obstetrics). All cell lines were maintained in DMEM with 10\% FBS. Prior to treatment the cells were incubated in phenol-red free DMEM supplemented with $20 \%$ charcoal stripped FBS overnight $(12-16 \mathrm{~h})$ followed by incubation with $5 \times 10^{-8}$ $\mathrm{M}$ estradiol, $1 \times 10^{-8} \mathrm{M}$ BSA-conjugated estradiol, and $1 \times$ 10-8 M G-1 for 4-18 h. Ethanol, phosphate-buffered saline (PBS), and dimethyl sulfoxide were used as the respective vehicle controls.

\section{Reverse transcriptase (RT) and real time RT-PCR}

Treated and untreated cells were rinsed with PBS and pelleted for RNA isolation. RNA was extracted using the RNeasy Midi kit (Qiagen Inc., Valencia, CA) according to the manufacturer's instructions. RNA purity and concentration were determined by spectrophotometry. cDNA was generated at a concentration equivalent to $25 \mathrm{ng} / \mu \mathrm{L}$ of RNA in a $20 \mu \mathrm{L}$ volume with random hexamers and Superscript II reverse transcriptase (Invitrogen Corporation). The PCR products were visualized on ethidium bromidestained 2\% agarose gels under UV light. Real-time PCR was carried out using the ABI Prism 7000 System. Tubulin was used as an internal control for normalization of each data point. Relative induction was calculated using the $2^{-}$ $\Delta \Delta \mathrm{CT}$ formula [16]. RNA was analyzed from three independent experiments.

\section{Western blotting}

Lysates were collected from treated and untreated cells in modified radioimmuno precipitation assay (RIPA) buffer containing EDTA and a protease inhibitor cocktail (Pierce Biotechnology, Rockford, IL) by standard methods. $40 \mu \mathrm{g}$ of protein was resolved by SDS-PAGE and transferred onto PVDF membranes. The membranes were subjected to immunodetection by incubation with primary antibody for STYK1 (1:500) and GPR30 (1:250). Equal protein loading was controlled by immunoblot of $\alpha$-tubulin (1:3000). The lysates from three independent experiments were analyzed. 


\section{Tissue panel and immunohistochemistry}

Formalin-fixed arrays of normal, benign, and malignant ovarian tissues were obtained from Pantomics Inc. (San Franscico, CA). The tissues were stained with a mouse monoclonal antibody for STYK1 and counterstained with hematoxylin by the Winship Cancer Institute Pathology Core Facility at Emory University. Each tissue section was assigned a score of 0 for none, 1 for weak, 2 for moderate, or 3 for strong STYK1 immunoreactivity. Scoring of the tissue sections was done by one of the authors without prior knowledge of the clinical parameters.

\section{Statistical analysis}

Statistical analyses were performed using the one-way ANOVA test in GraphPad Prism (San Diego, CA). The data are presented as mean \pm standard error.

\section{Results \\ Expression of STYKI in normal, benign, and malignant ovarian tissues}

Each normal ovarian tissue section was negative for STYK1 immunoreactivity (Fig. 1A). Although several of the benign ovarian tissue sections were positive for STYK1 immunoreactivity the staining intensity was weak (represented by the staining in normal tissue). The remaining benign ovarian tissues as well as one malignant ovary showed no STYK1 immunoreactivity. Many of the ovarian cancer tissue sections had weak STYK1 staining intensity as seen in the benign tissues, however, moderate and strong STYK1 staining intensity was seen only in the malignant ovarian tissues (i.e. endometroid adenocarcinomas). STYK1 immunoreactivity was cytoplasmic in every STYK1-positive ovarian tissue section (Fig. 1B).

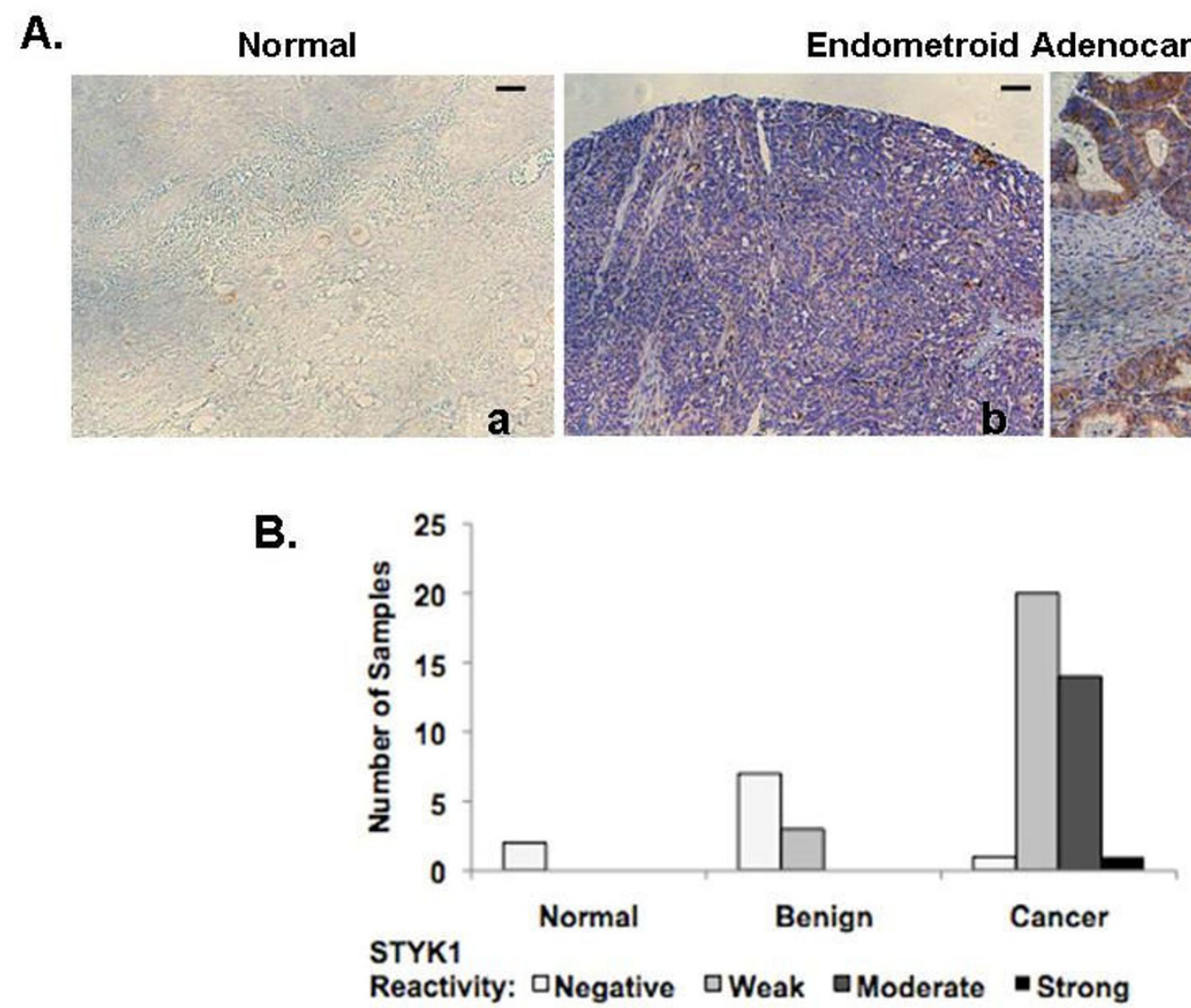

Figure I

STYKI Protein Expression is Associated with Ovarian Cancer. An ovarian tissue array (Pantomics, Inc.) was stained with STYKI antibody. (A) Each tissue section was assigned a score of 0 for no staining, I for weak, 2 for moderate, or 3 for strong STYKI reactivity. (B) STYKI localizes to the cytoplasm in malignant ovarian tissues. Representative of sections (20x magnification) immunohistochemical stains of normal and endometroid adenocarcinomas with anti-STYKI antibody shown, from left to right, no staining (a), weak (b), and moderate staining (c). 
A.

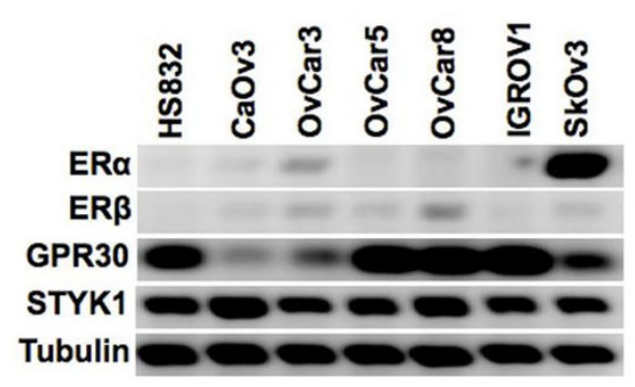

B.

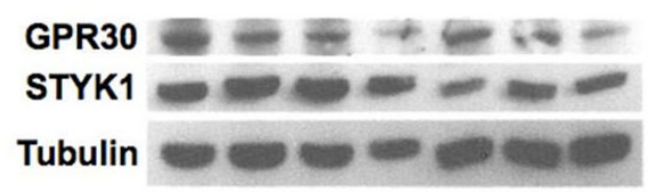

Figure 2

Ovarian tumor cell lines express STYKI and variably express estrogen receptors. HS832 is a benign ovarian cell line and the remaining cell lines were derived from ovarian cancer cell lines. (A) Ethidium bromide-induced fluorescence of RT-PCR amplification product from ovarian tumor RNA using primers for ER $\alpha$, ER $\beta$, GPR 30, and STYKI. Tubulin primers were used as a loading control; (B) STYKI and GPR30 protein expression. The western blot was stripped and re-probed with $\alpha$-tubulin antibody as a loading control.

\section{Expression of estrogen receptors and STYKI in ovarian cancer cell lines}

We detected ER- RNA expression in ovarian cancer cell lines SKOV3, CaOv3, and OvCar3 (Fig. 2A). Cell lines HS832, OvCar5, OvCar8 and IGROV had no detectable ER transcript. While ER expression was weak for most of the cell lines, no expression was detected in HS832 and IGROV1 cells. Every cell line expressed GPR30; however, strong expression was seen only in HS832, OvCar5, OvCar8, and IGROV1. GPR30 and STYK1 protein was detected at varying levels in each cell line (Fig. 2B).

\section{Estradiol and GPR30-specific G-I induce STYKI RNA but not protein expression in ovarian cancer cell lines}

RNA isolated from estradiol-treated HS832, OvCar5, OvCar8, and SkOv3 cells was analyzed by real time RTPCR with STYK1 primers. STYK1 expression increased significantly (p < 0.001) in HS832 cells (ER neg., ER- neg., GPR30 pos.) and decreased by almost half in the SkOv3 cells (ER pos., ER- pos., GPR30 pos.) after 18 hours in the presence of estradiol (Fig. 3A). While STYK1 expression decreased slightly in OvCar5 cells (ER neg., ER- pos., GPR30 pos.), there was no real change in expression in OvCar8 cells, which have the same ER expression profile.

HS832, OvCar5, and SkOv3 cells treated (16 hours) with G-1, a GPR30-specific ligand, showed an increase in
STYK1 expression relative to 16 hours estradiol-treated cells although the increase was only significant in OvCar5 cells ( $\mathrm{p}<0.001$; Fig. 3B). Conversely, STYK1 expression decreased slightly in G-1-treated OvCar8 cells. When the cells were treated with BSA-conjugated estradiol (E2B), which allows estradiol to interact with receptors on the cell membrane but prevents the molecule from entering the cell, STYK1 expression increased relative to estradiolinduced expression in OvCar5 cells but decreased in OvCar8 cells. There was no appreciable change in STYK1 expression in HS832 and SkOv3 cells. In contrast to STYK1 RNA expression, STYK1 protein expression levels were unaffected by estradiol, BSA-conjugated estradiol, and G-1 treatments, with the possible exception of OvCar5, where a slight increase in STYK1 was observed with E2B after 16 hours post-treatment (Fig. 4).

\section{Discussion and Conclusion}

STYK1 mRNA levels have been reported in human benign and/or malignant tissues, but the immunoreactivity of STYK1 has not been reported. Several reports demonstrate STYK1 mRNA expression in various normal tissues and STYK1 overexpression in breast and lung cancer tissues and cell lines, as well as in patients with acute leukemia [17-19]. Moreover, Moriai et. al reported high levels of STYK1 expression even in early stages of breast cancer. In this study, we demonstrated the presence of STYK1 immunoreactivity, in benign and malignant ovarian tissues and cell lines but not in normal ovarian tissue. Moreover, benign ovarian tissues displaying immunoreactivity for STYK1 displayed weak staining. Moderate and strong STYK1 staining was seen only in the high grade ovarian cancer tissues. This data suggests that STYK1 is associated with tumorigenic and malignant phenotypes in ovarian tissue. However, it should be noted that duplicates of only two normal tissue sections were analyzed in this study. With more samples this data should support the need for future studies to validate STYK1 as a potential prognostic tool for detecting multiple stages of ovarian carcinogenesis.

Our lab previously demonstrated that estradiol increases STYK1 mRNA levels in ER negative, ER positive MDA-MB 231 breast cancer cells [10]. In the current study estradiol downregulated STYK1 in OvCar5 cells expressing ER $\beta$ but not ER $\alpha$ but did not have a notable affect on STYK1 mRNA levels in OvCar8 cells, which have the same estrogen receptor expression profile (Fig. 3A). This might be due to the presence of higher levels of GPR30 downstream signaling proteins or EGFR/HER protein levels, which are involved in signaling through GPR30. This would be supported in the increase of STYK1 due to G1 treatment, OvCar5 versus OvCar8. It is notable that the level of GPR30 mRNA is not reflective of the relative levels of GPR30 protein. Further investigation into the mechanism 

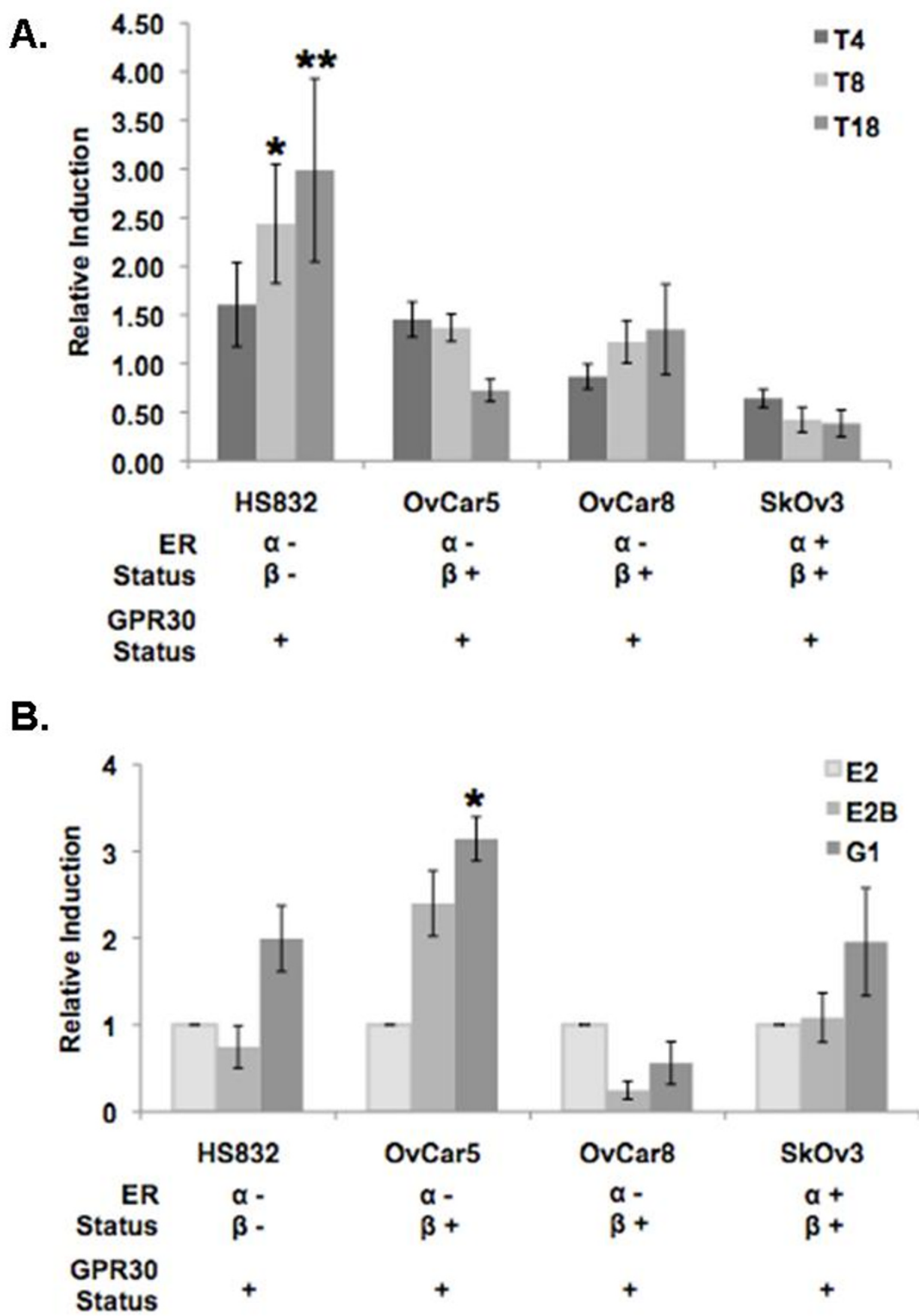

\section{Figure 3}

Estradiol and G-I induce STYKI RNA expression. Ovarian tumor cell lines were treated with vehicle, $5 \times 10^{-8} \mathrm{M}$ estradiol, I $\times 10^{-8} \mathrm{M}$ BSA-conjugated estradiol (E2B), and I $\times 10^{-8} \mathrm{M} \mathrm{G}$-I for 4-I8 h(T4, T8, TI8). cDNA equivalent to $25 \mathrm{ng} / \mu \mathrm{L}$ of RNA was generated and analyzed by real-time RT-PCR. Relative values were normalized to $\alpha$-tubulin and values were compared to the vehicle. Values are the mean of 3 independent experiments. (A) STYKI induction in estradiol-treated ovarian tumor cells relative to untreated cells for various intervals; (B) STYKI induction in E2-, E2B- and GI-treated cells relative to estradiol-induced STYKI expression following 18 hours. * and ** indicate values that are significantly different compared to the vehicle $(p<0.01$ and $p<0.001$, respectively). 
of GPR30 expression and regulation is underway. However, the higher ER $\beta$ levels in the OvCar8 cells could account for the difference in STYK1 regulation. Interestingly, the highest STYK1 induction was seen in the HS832 cells $(8 \mathrm{~h}, \mathrm{p}<0.01 ; 18 \mathrm{~h}, \mathrm{p}<0.001)$, which are ER and ER negative while the ER and ER positive SkOv3 cells had a marked reduction in STYK1 expression in response to estradiol treatment. This data suggests that there is an inverse relationship between estradiol-mediated STYK1 regulation and ER/ER expression. A similar observation was observed in MCF7, ER $\alpha$ positive, ER $\beta$ negative versus MDA-MB-231 which is ER $\alpha$ negative, ER $\beta$ positive [10]. ER was previously shown to downregulate the FN1 gene in ovarian cancer cells and ER $\beta$ expression is inversely correlated with tumorigenesis in ovarian cells $[11,20]$. Regulation of STYK1 expression in cells negative for ER and ER points to estradiol-mediated regulation through a nontraditional hormone receptor pathway, possibility GPR30.

GPR30, a novel estrogen receptor was recently reported to mediate changes in gene expression and growth in ovarian cancer cells treated with estradiol [15]. We showed that G-1, a GPR30-specific ligand, induced STYK1 at a higher level in the ovarian tumor cells than estradiol. A significant increase $(\mathrm{p}<0.001)$ in G-1-induced STYK1 expression was seen in OvCar5 cells, which do not express ER, the primary estradiol receptor, but expresses ER $\beta$ at very weak levels. In contrast, G-1 downregulated STYK1 in OvCar8 cells, which expressed no ER $\alpha$ and the highest ER $\beta$ levels of the analyzed cell lines. We speculate that STYK1 is a downstream target of estrogen-mediated GPR30 activation in ovarian cancer cells and that the affect of GPR30 on STYK1 expression is more pronounced in the absence of ER and ER. This difference in STYK1 regulation could be due to the loss preferential or competitive binding of estradiol to ER and/or ER . It is important to note that one study reported that estradiol does not activate GPR30 [21]. It would also imply that the affect ER and/or ER on GPR30-mediated regulation of STYK1 expression occurs through a mechanism other than competitive ligand binding.

The cellular localization of GPR30 is controversial. It has been reported to localize to the cell membrane and the endoplasmic reticulum membrane $[21,22]$. We addressed this issue; G-1-induced STYK1 expression was compared to that in cells treated with BSA-conjugated estradiol (E2B), which is too large to enter the cell. Therefore, any estradiol-induced STYK1 expression would occur through binding of estradiol to a cell membrane receptor. E2B induced STYK1 expression at a level similar to the estradiol induction in each cell line except OvCar5, where STYK1 expression doubled. However, E2B-induction was consistently lower than that seen in G-1-treated cells. This data supports localization of GPR30 to the cell membrane but does not controvert reports of its intracellular localization and provides further evidence of estradiol binding to GPR30.

Liu et. al reported tumorigenesis and metastasis of normal cells (NIH3T3 and BaF3) overexpressing STYK1 in nude mice [5]. Their group suggested that abnormal expression of STYK1 results in a constitutively active state caused by disruption of an inactive vs. active state equilibrium. However, we did not see an appreciable difference in total

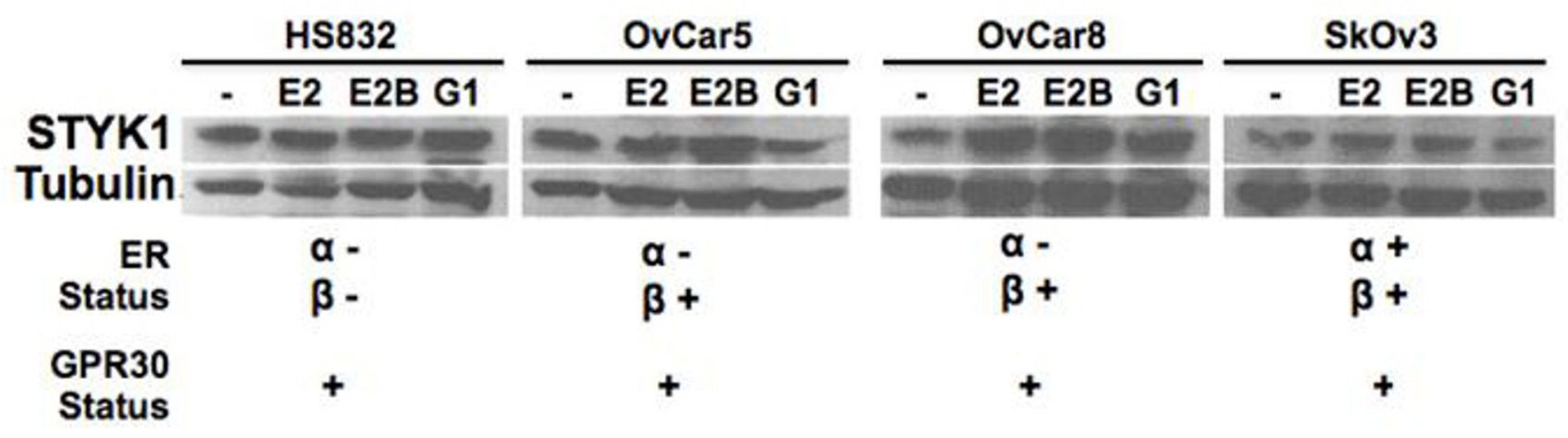

Figure 4

STYKI protein expression is unaffected by estradiol and G-I treatment. Ovarian tumor cell lines were treated with vehicle, $5 \times 10^{-8} \mathrm{M}$ estradiol, I × 10-8 M BSA-conjugated estradiol (E2B), and I $\times 10^{-8} \mathrm{M} \mathrm{G-I}$ for $4-18 \mathrm{~h}$ for $18 \mathrm{hours}$. Forty $\mu \mathrm{g}$ of protein lysate from the indicated cell lines were electrophoresed by $12.5 \%$ SDS PAGE and transferred to PVDF membrane. The blot was probed with anti-STYKI antibody then stripped and re-probed with $\alpha$-tubulin as a loading control. This is a representative blot from three independent experiments. * indicates a value that is significantly different compared to $5 \times 10^{-8} \mathrm{M}$ estradiol treatment $(p<0.00 \mathrm{I})$. 
STYK1 protein levels in cells treated with estradiol and G1 compared to untreated controls. It is possible that changes in STYK1 protein levels occur early in tumorigenesis and that estradiol does not further induce STYK1 overexpression. Nonetheless, studies show that STYK1 activity is regulated by phosphorylation and dephosphorylation of several tyrosine residues within exon $11[6,9]$. Therefore, both STYK1 and GPR30 might be model therapeutic targets for the development of more effective ovarian cancer treatments. These molecular targets may also be especially important in treating triple negative (ER negative, HER2 negative, progesterone receptor negative) breast cancers, which are often nonresponsive to standard chemotherapeutic medications that target traditional hormone receptors [10].

\section{Competing interests}

The authors declare that they have no competing interests.

\section{Authors' contributions}

KAJ carried out the molecular genetic studies, westerns, performed the statistical analysis, and drafted the manuscript. JH assisted and carried out the westerns. GO carried out analysis of immunohistochemistry. KSK conceived the study, and participated in its design and coordination. All authors read and approved the final manuscript.

\section{Acknowledgements}

Thanks to Neil Sidell for his comments and review of the manuscript. This work was supported in part by the Fellowships in Research and Science Teaching (FIRST) postdoctoral program at Emory University School of Medicine NIH KI2-GM000680 and NIH 5P60 MD000525-02.

\section{References}

I. Lingeman $\mathrm{CH}$ : Etiology of cancer of the human ovary: a review. J Natl Cancer Inst 1974, 53:1603-1618.

2. Scott M, McCluggage WG, Hillan KJ, Hall PA, Russell SE: Altered patterns of transcription of the septin gene, SEPT9, in ovarian tumorigenesis. Int J Cancer 2006, I I 8: I 325- I 329.

3. Smith T, Stein KD, Mehta CC, Kaw C, Kepner JL, Buskirk T, Stafford J, Baker F: The rationale, design, and implementation of the American Cancer Society's studies of cancer survivors. Cancer 2007, 109:1-12.

4. Wu F, Safe S: Differential activation of wild-type estrogen receptor alpha and C-terminal deletion mutants by estrogens, antiestrogens and xenoestrogens in breast cancer cells. j Steroid Biochem Mol Biol 2007, I 03: I-9.

5. Liu L, Yu XZ, Li TS, Song LX, Chen PL, Suo TL, Li YH, Wang SD, Chen $Y$, Ren $Y M$, et al:: A novel protein tyrosine kinase NOK that shares homology with platelet- derived growth factor/fibroblast growth factor receptors induces tumorigenesis and metastasis in nude mice. Cancer Res 2004, 64:3491-3499.

6. Li YH, Zhong S, Rong ZL, Ren YM, Li ZY, Zhang SP, Chang Z, Liu L: The carboxyl terminal tyrosine 417 residue of NOK has an autoinhibitory effect on NOK-mediated signaling transductions. Biochem Biophys Res Commun 2007, 356:444-449.

7. Ho SM: Estrogen, progesterone and epithelial ovarian cancer. Reprod Biol Endocrinol 2003, I:73.

8. Syed V, Ulinski G, Mok SC, Ho SM: Reproductive hormoneinduced, STAT3-mediated interleukin 6 action in normal and malignant human ovarian surface epithelial cells. J Natl Cancer Inst 2002, 94:617-629.

9. Chen Y, Li YH, Chen XP, Gong LM, Zhang SP, Chang ZJ, Zhang XF, Fu XY, Liu L: Point mutation at single tyrosine residue of novel oncogene NOK abrogates tumorigenesis in nude mice. Cancer Res 2005, 65:10838-10846.

10. Kimbro KS, Duschene K, Willard M, Moore JA, Freeman S: A novel gene STYKI/NOK is upregulated in estrogen receptor-alpha negative estrogen receptor-beta positive breast cancer cells following estrogen treatment. Mol Biol Rep 2008, 35:23-27.

II. Cunat S, Hoffmann P, Pujol P: Estrogens and epithelial ovarian cancer. Gynecol Oncol 2004, 94:25-32.

12. Roa BR, Slotman BJ: Action and counter-action of hormones in human ovarian cancer. Anticancer Res 1989, 9:1005-1007.

13. Backstrom T, Sanders D, Leask R, Davidson D, Warner P, Bancroft J: Mood, sexuality, hormones, and the menstrual cycle. II. Hormone levels and their relationship to the premenstrual syndrome. Psychosom Med 1983, 45:503-507.

14. Mahlck CG, Backstrom T, Kjellgren O: Androstenedione production by malignant epithelial ovarian tumors. Gynecol Oncol 1986, 25:217-222.

15. Albanito L, Madeo A, Lappano R, Vivacqua A, Rago V, Carpino A, Oprea TI, Prossnitz ER, Musti AM, Ando S, Maggiolini M: G proteincoupled receptor 30 (GPR30) mediates gene expression changes and growth response to I 7beta-estradiol and selective GPR30 ligand G-I in ovarian cancer cells. Cancer Res 2007, 67:1859-1866.

16. Yalcin A: Quantification of thioredoxin mRNA expression in the rat hippocampus by real-time PCR following oxidative stress. Acta Biochim Pol 2004, 5 I : I059- 065.

17. Moriai R, Kobayashi D, Amachika T, Tsuji N, Watanabe N: Diagnostic relevance of overexpressed NOK mRNA in breast cancer. Anticancer Res 2006, 26:4969-4973.

18. Amachika T, Kobayashi D, Moriai R, Tsuji N, Watanabe N: Diagnostic relevance of overexpressed mRNA of novel oncogene with kinase-domain (NOK) in lung cancers. Lung Cancer 2007, 56:337-340.

19. Kondoh T, Kobayashi D, Tsuji N, Kuribayashi K, Watanabe N: Overexpression of serine threonine tyrosine kinase I/novel oncogene with kinase domain mRNA in patients with acute leukemia. Exp Hematol 2009, 37:824-830.

20. O'Donnell AJ, Macleod KG, Burns DJ, Smyth JF, Langdon SP: Estrogen receptor-alpha mediates gene expression changes and growth response in ovarian cancer cells exposed to estrogen. Endocr Relat Cancer 2005, I 2:85 I-866.

21. Otto C, Fuchs I, Kauselmann G, Kern H, Zevnik B, Andreasen P, Schwarz G, Altmann H, Klewer M, Schoor M, et al.: GPR30 does not mediate estrogenic responses in reproductive organs in mice. Biol Reprod 2009, 80:34-4I.

22. Funakoshi T, Yanai A, Shinoda K, Kawano MM, Mizukami Y: G protein-coupled receptor 30 is an estrogen receptor in the plasma membrane. Biochem Biophys Res Commun 2006, 346:904-910.

\section{Publish with Bio Med Central and every scientist can read your work free of charge}

"BioMed Central will be the most significant development for disseminating the results of biomedical research in our lifetime. "

Sir Paul Nurse, Cancer Research UK

Your research papers will be:

- available free of charge to the entire biomedical community

- peer reviewed and published immediately upon acceptance

- cited in PubMed and archived on PubMed Central

- yours - you keep the copyright

Submit your manuscript here:

http://www.biomedcentral.com/info/publishing_adv.asp 